\title{
Correlation of blood glucose, serum chemerin and insulin resistance with NAFLD in patients with type 2 diabetes mellitus
}

\author{
ZHENGJUN ZHANG $^{1 *}$, JIJUN WANG $^{2 *}$ and HONGMEI WANG ${ }^{2}$ \\ ${ }^{1}$ Department of Endocrinology, The Affiliated Hospital of Jining Medical University, Jining, Shandong 272000; \\ ${ }^{2}$ Department of Internal Medicine, Jining Municipal Government Hospital, Jining, Shandong 272100, P.R. China
}

Received August 24, 2017; Accepted December 11, 2017

DOI: 10.3892/etm.2018.5753

\begin{abstract}
Non-alcoholic fatty liver disease (NAFLD) is a form of clinical syndrome characterized by the fatty degeneration in liver histology and should be further investigated. The aim of the study was to investigate the effects of blood glucose, serum chemerin and insulin resistance on non-alcoholic fatty liver disease (NAFLD) in patients with type 2 diabetes mellitus to provide a basis for the prevention and treatment thereof. In total, 300 patients with type 2 diabetes mellitus treated and admitted into the Endocrinology Department of our hospital from June 2015 to June 2017 were enrolled and divided into the simple type 2 diabetes mellitus (group A) and concurrent NAFLD (group B) groups. The sex, age, body mass index (BMI), blood pressure, blood biochemical indexes and chemerin level were compared between the two groups. The patients in group B were further divided into the mild fatty liver (group B1), moderate fatty liver (group B2) and severe fatty liver (group B3) groups. The sex, age, BMI blood pressure, blood biochemical indexes and chemerin level were also compared among the three groups. Finally, the risk factors of type 2 diabetes mellitus complicated by NAFLD were analyzed via logistic regression. The BMI, fasting plasma glucose (FPG), 2 h post-prandial plasma glucose (2hPG), triglyceride (TG), total cholesterol (TC), low-density lipoprotein cholesterol (LDL-c), alanine aminotransferase (ALT), fasting insulin (FINS), homeostasis model assessment of insulin resistance (HOMA-IR) and HOMA- $\beta$ indexes and serum chemerin level in group B were significantly higher than those in group $\mathrm{A}(\mathrm{P}<0.05$ or $\mathrm{P}<0.01)$. Notably, the aggravation of NAFLD, the aforementioned indexes were obviously
\end{abstract}

Correspondence to: Dr Hongmei Wang, Department of Internal Medicine, Jining Municipal Government Hospital, 20 Hongxing Middle Road, Rencheng, Jining, Shandong 272100, P.R. China

E-mail: jyfy0123@126.com

*Contributed equally

Key words: type 2 diabetes mellitus, nonalcoholic fatty liver disease, insulin resistance, blood glucose, chemerin increased $(\mathrm{P}<0.05$ or $\mathrm{P}<0.01)$. The regression analysis revealed that BMI, FPG, TC, LDL-c, FINS, HOMA-IR and chemerin were risk factors of concurrent NAFLD. Thus, type 2 diabetes mellitus complicated by NAFLD is closely associated with severe glucose-lipid metabolism disorder and insulin resistance, and BMI, FPG, TC, LDL-c, FINS, HOMA-IR and chemerin constitute risk factors of concurrent NAFLD.

\section{Introduction}

Non-alcoholic fatty liver disease (NAFLD) is a kind of clinical syndrome characterized by fatty degeneration in liver histology. NAFLD is closely related to the metabolic diseases, such as type 2 diabetes mellitus, insulin resistance and obesity, of which insulin resistance is the common pathophysiological basis (1). Previous findings showed that NAFLD may increase the risks of type 2 diabetes mellitus and its complications in patients (2). Additional findings revealed that the prevalence and mortality rates of NAFLD in patients with type 2 diabetes mellitus were also significantly higher (3). According to the National Health and Nutrition Examination Survey in the United States, the independent risk factors of NAFLD include insulin resistance and type 2 diabetes mellitus, which collectively contribute towards an increase in the mortality risks of NAFLD (4). Chemerin is a kind of newly-discovered adipocytokine, which is closely associated with the occurrence of metabolic diseases, such as type 2 diabetes mellitus, obesity and metabolic syndrome $(5,6)$. The present study aimed to investigate the correlation of blood glucose, serum chemerin and insulin resistance with concurrent NAFLD in patients with type 2 diabetes mellitus by comparing changes in blood glucose, serum chemerin and insulin resistance, between patients with type 2 diabetes mellitus and patients with type 2 diabetes mellitus complicated by NAFLD. Furthermore, the present study also focused on the changes in blood glucose, serum chemerin and insulin resistance, among patients with NAFLD in different degrees in order to provide a basis for the comprehensive treatment and prevention of associated pathological states at moderate NAFLD stage.

The prime advantage of the present study was the novel reference standards in terms of blood glucose, serum chemerin and insulin resistance for the timely assessment of NAFLD at variable stages (mild, moderate and severe). NAFLD at mild or moderate stages is more of lifestyle prone pathological state. 
At the mild stage of NAFLD, it is likely that the disease may be reversed through a proper diet plan and physical activities. In this manner, the prevention of NAFLD at mild/moderate levels may indirectly help to prevent more deadly pathological states such as metabolic syndrome, diabetes and cardiac disorders. Thus, the study opens new gates of rapid assessment of NAFLD at the mild/moderate stage to ensure patients are adequately informed regarding sever consequences of the disease in future.

\section{Materials and methods}

General materials. In total, 300 patients with type 2 diabetes mellitus treated and admitted into the Endocrinology Department of the The Affiliated Hospital of Jining Medical University (Shandong, China) from June 2015 to June 2017 were included in the present study. The diagnostic criteria of type 2 diabetes mellitus were based on the WHO diagnostic criteria of diabetes mellitus in 1998 (7), i.e., fasting blood glucose $\geq 7.0 \mathrm{mmol} / \mathrm{l}$, or typical symptoms of diabetes mellitus with random blood glucose $\geq 11.1 \mathrm{mmol} / 1$, or $2 \mathrm{~h}$ blood glucose in glucose tolerance test $\geq 11.1 \mathrm{mmol} / 1$. All the patients enrolled were aged 30-70 years. Exclusion criteria for the study were: i) patients with type 1 diabetes mellitus, gestational diabetes, diabetic ketoacidosis, hyperglycemic hyperosmolar status, severe peripheral neuropathy, severe peripheral vascular disease, acute infection or other severe diabetic complications; ii) patients with viral hepatitis, alcoholic hepatitis, drug-induced hepatitis or autoimmune hepatitis; iii) patients with hepatic or renal dysfunction, cancer or mental disease. The study was approved by the Ethics Committee of The Affiliated Hospital of Jining Medical University (Shandong, China) and written informed consents were signed by the patients and/or guardians.

Grouping. According to whether they were complicated by NAFLD, the 300 patients were divided into the simple type 2 diabetes mellitus (group $\mathrm{A}, \mathrm{n}=80$ ) and concurrent NAFLD (group $\mathrm{B}, \mathrm{n}=220$ ) groups. Diagnostic criteria of NAFLD were (8): i) patients with a history of heavy drinking, or weekly alcohol intake $<140 \mathrm{~g}$ in males and $<70 \mathrm{~g}$ in females; ii) patients with viral, drug-induced or autoimmune hepatitis, cirrhosis, hepatolenticular degeneration, total parenteral nutrition or other disease that induces fatty liver disease; iii) pathological changes in liver biopsy meet the pathological criteria of fatty liver disease. According to the color Doppler imaging of fatty liver, the NAFLD patients were subdivided into the mild fatty liver (group B1), moderate fatty liver (group B2) and severe fatty liver (group B3) groups. The specific ultrasound findings were: i) the near-field echo in hepatic region was enhanced in a diffused mode compared with kidney and spleen, while the far-field echo was attenuated; ii) the edge angle of liver was blunt with mild to moderate enlargement; iii) intrahepatic duct had an unclear structure; iv) intrahepatic blood flow signal was reduced, but the intrahepatic blood vessel direction remained normal; and v) the echoes in the hepatic diaphragm and right lobe membrane were uneven. Patients with the manifestation i) and any one of manifestations ii-iv) were diagnosed as mild fatty liver; those with the manifestation i) and any two of manifestations ii-iv) were diagnosed as moderate fatty liver; those with the manifestations i) and v) and any two of the manifestations ii-iv) were diagnosed as severe fatty liver. Finally, there were 84 cases in group B1, 75 cases in group B2 and 61 cases in group B3.

Collection of clinical data. The age, sex, past medical history, medication history, height, body weight and blood pressure of patients were collected. In addition, the body mass index was calculated $\left[\mathrm{BMI}=\right.$ body weight $(\mathrm{kg}) /$ height $\left.\left(\mathrm{m}^{2}\right)\right]$.

Collection of blood samples. Three samples of fasting venous blood were collected from the patients in the early morning at 2 days ( $3 \mathrm{ml} /$ copy). Two of the three samples received a biochemical analysis and fasting insulin (FINS) detection in the Laboratory Department of our hospital. Serum was separated from an additional 1 sample and stored in a refrigerator at $-80^{\circ} \mathrm{C}$ for the detection of chemerin. Venous blood ( $3 \mathrm{ml}$ ) was collected at $2 \mathrm{~h}$ after meals for the detection of $2 \mathrm{~h}$ post-prandial plasma glucose (2hPG).

Detection of indexes. The biochemical indexes, FINS and 2hPG were detected using the Roche Cobas-8000 automatic biochemical analyzer (Roche Diagnostics, Basel, Switzerland). The serum chemerin level was measured using the ELISA kit (R\&D Systems, Minneapolis, MN, USA). After the loading and treatment according to the instructions of kit, the optical density (OD) value at $450 \mathrm{~nm}$ was measured using the continuous wavelength multi-functional microplate reader (Tecan, Grödig, Austria), the standard curve was drawn, and the chemerin content in each sample was calculated. The insulin resistance of patients was evaluated via the homeostasis model assessment of insulin resistance (HOMA-IR): HOMA-IR = fasting plasma glucose $(\mathrm{FPG})(\mathrm{mmol} / \mathrm{l}) \times \mathrm{FINS}$ $(\mathrm{mIU} / \mathrm{l}) / 22.5$. Pancreatic $\beta$-cell function was evaluated via HOMA- $\beta$ : HOMA- $\beta=$ FINS (mIU/l) x 20/[FPG (mmol/l)-3.5].

Statistical analysis. Statistical analysis was performed using SPSS 19.0 software (IBM Corp., New York, NY, USA). Measurement data were presented as mean \pm SD. An independent-samples t-test was used for the comparison between two groups, and Kruskal-Wallis test for the comparisons among the groups. The Chi-square test was used for the comparisons of enumeration data. The correlation analysis of risk factors of concurrent NAFLD was performed via logistic regression analysis. $\mathrm{P}<0.05$ was considered to indicate a statistically significant difference.

\section{Results}

Comparisons of clinical data and blood biochemical indexes between groups A and B. BMI, FPG, 2hPG, triglyceride (TG), total cholesterol (TC), low-density lipoprotein cholesterol (LDL-c), and alanine aminotransferase (ALT) in group B were significantly higher than those in group $\mathrm{A}$, and the differences were statistically significant $(\mathrm{P}<0.05$ or $\mathrm{P}<0.01)$. There were no significant differences in sex ratio, age, systolic pressure, diastolic pressure, high-density lipoprotein cholesterol (HDL-c) and aspartate aminotransferase (AST) between the two groups $(\mathrm{P}>0.05)$ (Table I). 
Table I. Comparisons of clinical data and blood biochemical indexes between groups A and B.

\begin{tabular}{lcc}
\hline Item & Group A & Group B \\
\hline Sex (male/female) & $49 / 31$ & $136 / 84$ \\
Age & $42.56 \pm 5.03$ & $43.21 \pm 6.47$ \\
BMI & $24.49 \pm 2.89$ & $26.77 \pm 2.65^{\mathrm{a}}$ \\
Systolic pressure & $123.20 \pm 17.23$ & $127.04 \pm 20.66$ \\
Diastolic pressure & $78.13 \pm 9.41$ & $79.65 \pm 10.82$ \\
FPG & $8.55 \pm 3.28$ & $12.93 \pm 3.50^{\mathrm{b}}$ \\
2hPG & $12.81 \pm 4.16$ & $16.68 \pm 4.24^{\mathrm{a}}$ \\
TG & $2.56 \pm 1.05$ & $3.44 \pm 0.94^{\mathrm{a}}$ \\
TC & $4.72 \pm 1.20$ & $5.81 \pm 1.43^{\mathrm{a}}$ \\
LDL-c & $2.48 \pm 0.83$ & $3.53 \pm 0.77^{\mathrm{b}}$ \\
HDL-c & $1.20 \pm 0.32$ & $1.06 \pm 0.28$ \\
ALT & $26.55 \pm 8.08$ & $33.42 \pm 7.69^{\mathrm{a}}$ \\
AST & $22.31 \pm 9.74$ & $27.50 \pm 8.63$ \\
\hline
\end{tabular}

${ }^{\mathrm{a}} \mathrm{P}<0.05,{ }^{\mathrm{b}} \mathrm{P}<0.01$, compared with group $\mathrm{A}$.

Table II. Comparisons of pancreatic $\beta$-cell function and insulin resistance between groups $\mathrm{A}$ and $\mathrm{B}$.

\begin{tabular}{lrr}
\hline Item & \multicolumn{1}{c}{ Group A } & \multicolumn{1}{c}{ Group B } \\
\hline FINS & $12.57 \pm 5.34$ & $15.21 \pm 5.81^{\mathrm{a}}$ \\
HOMA-IR & $4.80 \pm 1.86$ & $8.72 \pm 3.03^{\mathrm{b}}$ \\
HOMA- $\beta$ & $48.72 \pm 9.29$ & $32.38 \pm 8.62^{\mathrm{b}}$ \\
\hline
\end{tabular}

${ }^{\mathrm{a}} \mathrm{P}<0.05,{ }^{\mathrm{b}} \mathrm{P}<0.01$, compared with group $\mathrm{A}$.

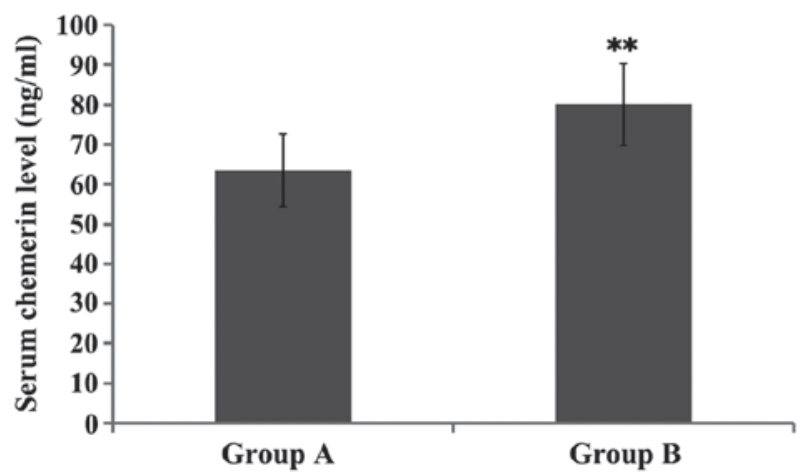

Figure 1. Comparison of serum chemerin level between groups A and B. ${ }^{* * *} \mathrm{P}<0.01$, compared with group A.

Comparisons of pancreatic $\beta$-cell function and insulin resistance between groups $A$ and $B$. FINS, HOMA-IR and HOMA- $\beta$ in group $B$ were significantly higher than those in group $\mathrm{A}$, and the differences were statistically significant $(\mathrm{P}<0.05$ or $\mathrm{P}<0.01)$ (Table II).

Comparison of serum chemerin level between groups A and $B$. The serum chemerin level in group $\mathrm{B}$ was significantly
Table III. Comparisons of clinical data and blood biochemical indexes among patients with fatty liver in different degrees.

\begin{tabular}{lccc}
\hline Item & Group B1 & Group B2 & Group B3 \\
\hline Sex (male/female) & $52 / 32$ & $46 / 29$ & $38 / 23$ \\
Age & $42.83 \pm 6.58$ & $43.36 \pm 6.13$ & $43.45 \pm 6.69$ \\
BMI & $24.81 \pm 2.77$ & $26.63 \pm 2.41^{\mathrm{a}}$ & $28.89 \pm 2.76^{\mathrm{b}, \mathrm{c}}$ \\
Systolic pressure & $125.62 \pm 21.63$ & $126.45 \pm 19.32$ & $129.03 \pm 21.00$ \\
Diastolic pressure & $77.66 \pm 11.48$ & $81.07 \pm 10.99$ & $80.24 \pm 9.98$ \\
FPG & $10.07 \pm 3.09$ & $13.10 \pm 3.77^{\mathrm{a}}$ & $15.62 \pm 3.65^{\mathrm{b}, \mathrm{c}}$ \\
2hPG & $13.50 \pm 4.02$ & $17.56 \pm 4.33^{\mathrm{a}}$ & $18.97 \pm 4.35^{\mathrm{a}}$ \\
TG & $3.15 \pm 1.00$ & $3.50 \pm 0.85^{\mathrm{a}}$ & $3.63 \pm 0.98^{\mathrm{a}}$ \\
TC & $4.94 \pm 1.26$ & $5.62 \pm 1.50^{\mathrm{a}}$ & $6.88 \pm 1.52^{\mathrm{b}, \mathrm{c}}$ \\
LDL-c & $2.71 \pm 0.85$ & $3.47 \pm 0.74^{\mathrm{b}}$ & $4.42 \pm 0.73^{\mathrm{b}, \mathrm{d}}$ \\
HDL-c & $1.14 \pm 0.27$ & $1.04 \pm 0.25$ & $1.01 \pm 0.31$ \\
ALT & $29.08 \pm 7.44$ & $32.71 \pm 7.70$ & $38.46 \pm 7.94^{\mathrm{a}, \mathrm{b}}$ \\
AST & $25.90 \pm 8.59$ & $27.19 \pm 8.42$ & $29.42 \pm 8.87$ \\
\hline
\end{tabular}

${ }^{\mathrm{a}} \mathrm{P}<0.05,{ }^{\mathrm{b}} \mathrm{P}<0.01$, compared with group $\mathrm{B} 1 ;{ }^{\mathrm{c}} \mathrm{P}<0.05,{ }^{\mathrm{d}} \mathrm{P}<0.01$, compared with group B2.

increased compared with that in group $\mathrm{A}$, and the difference was statistically significant $(\mathrm{P}<0.01)$ (Fig. 1).

Comparisons of clinical data and blood biochemical indexes in patients with fatty liver in different degrees. Patients in group B were divided and compared according to the different degrees of fatty liver. The results showed that the BMI, FPG, 2hPG, TG, TC and LDL-2 in groups B2 and $\mathrm{B} 3$ were significantly increased compared with those in group B1, and ALT in group B3 was significantly increased, with the differences being statistically significant $(\mathrm{P}<0.05$ or $\mathrm{P}<0.01)$. The BMI, FPG, TC, LDL-c and ALT in group B3 were obviously higher than those in group B2, and the differences were statistically significant $(\mathrm{P}<0.05$ or $\mathrm{P}<0.01)$ (Table III).

Comparisons of pancreatic $\beta$-cell function and insulin resistance among patients with fatty liver in different degrees. Patients in group B were divided and compared according to the different degrees of fatty liver. The results showed that the FINS and HOMA-IR in groups B2 and B3 were significantly increased, but HOMA- $\beta$ was significantly decreased compared with those in group B1, and the differences were statistically significant $(\mathrm{P}<0.05$ or $\mathrm{P}<0.01)$. HOMA-IR in group $\mathrm{B} 3$ was obviously higher than that in group B2, and the difference was statistically significant $(\mathrm{P}<0.01)$ (Table IV).

Comparison of serum chemerin level among patients with fatty liver in different degrees. Patients in group B were divided and compared according to the different degrees of fatty liver. The results showed that the serum chemerin levels in groups B2 and B3 were significantly higher than that in group B1, and the serum chemerin level in group B3 was obviously higher than that in group B2, and the differences were statistically significant $(\mathrm{P}<0.05$ or $\mathrm{P}<0.01)$ (Fig. 2). 
Table IV. Comparisons of pancreatic $\beta$-cell function and insulin resistance among patients with fatty liver in different degrees.

\begin{tabular}{lrrc}
\hline Item & Group B1 & Group B2 & Group B3 \\
\hline FINS & $12.83 \pm 5.17$ & $15.06 \pm 5.52^{\mathrm{a}}$ & $17.75 \pm 6.73^{\mathrm{a}}$ \\
HOMA-IR & $5.75 \pm 2.84$ & $8.77 \pm 3.11^{\mathrm{a}}$ & $12.30 \pm 3.15^{\mathrm{b}, \mathrm{c}}$ \\
HOMA- $\beta$ & $39.04 \pm 9.30$ & $31.38 \pm 8.41^{\mathrm{a}}$ & $29.28 \pm 8.18^{\mathrm{a}}$
\end{tabular}

${ }^{\mathrm{a}} \mathrm{P}<0.05$, ${ }^{\mathrm{b}} \mathrm{P}<0.01$, compared with group $\mathrm{B} 1$; ${ }^{\mathrm{c}} \mathrm{P}<0.01$, compared with group B2.

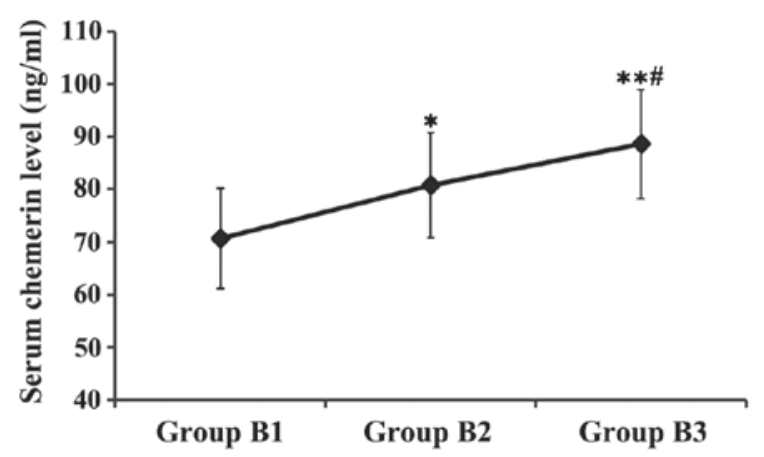

Figure 2. Comparison of serum chemerin level in patients with fatty liver in different degrees. ${ }^{*} \mathrm{P}<0.05,{ }^{* *} \mathrm{P}<0.01$, compared with group $\mathrm{B} 1 ;{ }^{*} \mathrm{P}<0.05$, compared with group B2.

Table V. Logistic regression analysis of risk factors of concurrent NAFLD.

\begin{tabular}{lccccc}
\hline $\begin{array}{l}\text { Independent } \\
\text { variable }\end{array}$ & B-value & Wald & P-value & OR & $\begin{array}{c}95 \% \text { confidence } \\
\text { interval (CI) }\end{array}$ \\
\hline BMI & 0.83 & 4.12 & 0.035 & 1.15 & $0.78-1.66$ \\
FPG & 1.12 & 7.66 & 0.004 & 2.87 & $1.23-5.64$ \\
TC & 0.72 & 3.44 & 0.042 & 0.95 & $0.67-1.50$ \\
LDL-c & 0.86 & 4.30 & 0.029 & 1.24 & $0.85-1.82$ \\
FINS & 1.05 & 6.53 & 0.010 & 2.12 & $1.11-4.97$ \\
HOMA-IR & 1.25 & 8.81 & 0.001 & 3.06 & $1.34-6.11$ \\
Chemerin & 0.78 & 3.81 & 0.038 & 1.02 & $0.71-1.59$ \\
\hline
\end{tabular}

Logistic regression analysis of risk factors of concurrent NAFLD. The risk factors were analyzed via binary logistic regression with the fatty liver as a dependent variable and with the sex, age, BMI, systolic pressure, diastolic pressure, FPG, 2hPG, TG, TC, LDL-c, HDL-c, ALT, AST, FINS, HOMA-IR, HOMA- $\beta$ and chemerin as the independent variables. The results revealed that BMI, FPG, TC, LDL-c, FINS, HOMA-IR and chemerin were independent risk factors of type 2 diabetes mellitus complicated by NAFLD (Table V).

\section{Discussion}

Non-alcoholic fatty liver disease (NAFLD) mainly occurs in the liver lobules, manifested as the fatty degeneration of liver cells and fatty accumulation of liver (8). The current incidence rate of NAFLD is up to 5-40\% in Asian countries, while it is up to $24-42 \%$ in Western countries, becoming a factor seriously harming the public health worldwide (9). NAFLD is a pathological process of excessive fatty accumulation caused by a variety of factors. At present, the pathogenesis of NAFLD remains unclear, and most scholars believe that its pathogenesis is related to the 'two-hit theory' (10). It is often believed that the action of heredity, drug, diabetes mellitus, obesity, lipid metabolism disorders and other factors leads to the excessive secretion of insulin, causing insulin resistance and resulting in increased fat in liver and mitochondrial dysfunction in liver cells, ultimately causing the first hit. Subsequently, the fatty degeneration of liver cell is induced, which decreases the cell viability and increases the intracellular oxidative metabolites, leading to the liver cell oxidative stress, ultimately causing the second hit. Finally, a series of inflammatory responses, necrosis and fibrosis occur in liver cells $(11,12)$.

Previous findings have shown that up to $50 \%$ of patients with type 2 diabetes mellitus are complicated by NAFLD, whereas the incidence rate of NAFLD is as high as $100 \%$ in patients with diabetes mellitus complicated by obesity (13). From the perspective of NAFLD, at least one third of NAFLD patients are complicated by diabetes mellitus and NAFLD can induce or aggravate insulin resistance in patients (14). It was found in a follow-up observation for patients with type 2 diabetes mellitus that NAFLD can increase the risk of cardiovascular diseases 2-fold, and it is speculated that NAFLD is also an independent risk factor of cardiovascular disease in patients with type 2 diabetes mellitus (15). Another study also found that NAFLD can increase the risk of complications, such as diabetic nephropathy and diabetic retinopathy, and it can be used as an independent risk factor (16). In this study, it was also found that $73 \%$ of patients with type 2 diabetes mellitus are complicated by NAFLD; thus, NAFLD is closely associated with the type 2 diabetes mellitus.

The close association between NAFLD and type 2 diabetes mellitus lies in that they have a common pathogenesis, insulin resistance (17). NAFLD can reduce the inhibitory effect of insulin in the body on endogenous glucose production, and reduce the body's insulin sensitivity, making it difficult to control blood glucose in patients with type 2 diabetes mellitus (18). In a study involving 2,839 patients with type 2 diabetes mellitus, the level of glycosylated hemoglobin in patients complicated by NAFLD was significantly increased compared with that in patients with simple diabetes mellitus, and the proportion of patients using insulin was also significantly increased (19). Kelley et al (20) identified that the HOMA-IR level in patients with type 2 diabetes mellitus complicated by NAFLD is significantly higher than that in patients with simple type 2 diabetes mellitus, and it can be seen that more serious insulin resistance exists in type 2 diabetes mellitus complicated by NAFLD. In this study, BMI, FPG, 2hPG, TG, TC, LDL-c, FINS and HOMA-IR in patients with type 2 diabetes mellitus complicated by NAFLD were significantly increased compared with those in patients with simple type 2 diabetes mellitus, whereas the pancreatic $\beta$-cell function was significantly decreased. The higher the levels of BMI, FPG, 2hPG, TG, TC, LDL-c, FINS and HOMA-IR were and the lower the pancreatic $\beta$-cell function was, the more severe was the NAFLD. Logistics regression analysis further confirmed that BMI, FPG, TC, LDL-c, FINS 
and HOMA-IR were independent risk factors of type 2 diabetes mellitus complicated by NAFLD.

Chemerin is a newly-discovered adipocytokine with a relative molecular mass of 16,000, and consisting of 163 amino acid residues, which is expressed in white adipose tissues, liver, lung and kidney (21). Chemerin can affect the insulin receptor signaling pathway, lead to insulin resistance or aggravate the body's original insulin resistance (5). Lehrke et al (22) revealed that the level of chemerin is increased in adipocytes, which can activate serine/threonine kinases, thereby reducing the tyrosine phosphorylation, inhibiting the translocation of glucose 4 , and causing insulin resistance in adipocytes. Zhuang et al (23) found that the serum chemerin level in NAFLD patients was significantly higher than that in healthy individuals, but was significantly decreased following treatment with metformin, suggesting that the occurrence of NAFLD is closely associated with the serum chemerin level, as well as insulin resistance. Another study on a Caucasian population found that the serum metabolin level was significantly increased in populations with risk factors of metabolic syndrome (24). In this study, it was found that the level of serum chemerin in patients with type 2 diabetes mellitus complicated by NAFLD was significantly increased compared with that in patients with simple type 2 diabetes mellitus, and the higher the serum chemerin level, the more severe the NAFLD was. Logistics regression analysis also further confirmed that chemerin was an independent risk factor of type 2 diabetes mellitus complicated by NAFLD.

The present study concludes that chemerin is a novel molecule that is useful in the timely assessment of NAFLD as it was clearly observed that, the higher the serum chemerin level was, the more sever NAFLD was. Timely assessment of NAFLD at a mild or moderate stage was reversed by appropriate changes in the lifestyle of the patients such as physical activities and proper diet. The reversal of NAFLD in this manner could in turn indirectly prevent the occurrence of deadly pathological states including type 2 diabetes. To the best of our knowledge, this is the first study to offer a novel form of assessment of NAFLD at the mild stage. Future studies with higher sample size are required for more concrete conclusions.

\section{Competing interests}

The authors declare that they have no competing interests.

\section{References}

1. Fruci B, Giuliano S, Mazza A, Malaguarnera R and Belfiore A Nonalcoholic fatty liver: A possible new target for type 2 diabetes prevention and treatment. Int J Mol Sci 14: 22933-22966, 2013.

2. Mavrogiannaki AN and Migdalis IN: Nonalcoholic fatty liver disease, diabetes mellitus and cardiovascular disease: Newer data. Int J Endocrino 2013: 450639, 2013.

3. Targher G, Bertolini L, Poli F, Rodella S, Scala L, Tessari R, Zenari L and Falezza G: Nonalcoholic fatty liver disease and risk of future cardiovascular events among type 2 diabetic patients. Diabetes 54: 3541-3546, 2005.

4. Stepanova M, Rafiq N and Younossi ZM: Components of metabolic syndrome are independent predictors of mortality in patients with chronic liver disease: A population-based study. Gut 59: 1410-1415, 2010.

5. Bozaoglu K, Bolton K, McMillan J, Zimmet P, Jowett J, Collier G, Walder K and Segal D: Chemerin is a novel adipokine associated with obesity and metabolic syndrome. Endocrinology 148: 4687-4694, 2007.
6. Chu SH, Lee MK, Ahn KY, Im JA, Park MS, Lee DC, Jeon JY and Lee JW: Chemerin and adiponectin contribute reciprocally to metabolic syndrome. PLoS One 7: e34710, 2012.

7. Alberti KG and Zimmet PZ: Definition, diagnosis and classification of diabetes mellitus and its complications. Part 1: diagnosis and classification of diabetes mellitus provisional report of a WHO consultation. Diabet Med 15: 539-553, 1998.

8. Macavei B, Baban A and Dumitrascu DL: Psychological factors associated with NAFLD/NASH: A systematic review. Eur Rev Med Pharmacol Sci 20: 5081-5097, 2016.

9. Amarapurkar DN, Hashimoto E, Lesmana LA, Sollano JD, Chen PJ and Goh KL; Asia-Pacific Working Party on NAFLD: How common is non-alcoholic fatty liver disease in the AsiaPacific region and are there local differences? J Gastroenterol Hepatol 22: 788-793, 2007.

10. Day CP and James OF: Steatohepatitis: A tale of two 'hits'? Gastroenterology 114: 842-845, 1998.

11. Kim CH and Younossi ZM: Nonalcoholic fatty liver disease: A manifestation of the metabolic syndrome. Cleve Clin J Med 75: 721-728, 2008.

12. Moreno Sánchez D: Pathogenesis of primary nonalcoholic fatty liver disease. Med Clin (Barc) 124: 668-677, 2005 (In Spanish).

13. Chitturi S, Wong VW and Farrell G: Nonalcoholic fatty liver in Asia: Firmly entrenched and rapidly gaining ground. J Gastroenterol Hepatol 26 (Suppl 1): 163-172, 2011.

14. Pacifico L, Nobili V, Anania C, Verdecchia P and Chiesa C: Pediatric nonalcoholic fatty liver disease, metabolic syndrome and cardiovascular risk. World J Gastroenterol 17: 3082-3091, 2011.

15. Targher G, Valbusa F, Bonapace S, Bertolini L, Zenari L, Rodella S, Zoppini G, Mantovani W, Barbieri E and Byrne CD: Non-alcoholic fatty liver disease is associated with an increased incidence of atrial fibrillation in patients with type 2 diabetes. PLoS One 8: e57183, 2013.

16. Targher G, Bertolini L, Rodella S, Zoppini G, Lippi G, Day C and Muggeo M: Non-alcoholic fatty liver disease is independently associated with an increased prevalence of chronic kidney disease and proliferative/laser-treated retinopathy in type 2 diabetic patients. Diabetologia 51: 444-450, 2008.

17. Jorgensen RA: Nonalcoholic fatty liver disease. Gastroenterol Nurs 26: 150-155, 2003.

18. Azam G, Alam S, Hasan SKMN, Alam SMNE, Kabir J and Alam AKMK: Insulin resistance in nonalcoholic fatty liver disease. Experience from Bangladesh. Bangladesh Critical Care Journal 4: 86, 2016. doi: org/10.3329/bccj.v4i2.30022.

19. Targher G, Bertolini L, Padovani R, Rodella S, Tessari R, Zenari L, Day C and Arcaro G: Prevalence of nonalcoholic fatty liver disease and its association with cardiovascular disease among type 2 diabetic patients. Diabetes Care 30: 1212-1218, 2007.

20. Kelley DE, McKolanis TM, Hegazi RA, Kuller LH and Kalhan SC: Fatty liver in type 2 diabetes mellitus: Relation to regional adiposity, fatty acids, and insulin resistance. Am J Physiol Endocrinol Metab 285: E906-E916, 2003.

21. Peng L, Yu Y, Liu J, Li S, He H, Cheng N and Ye RD: The chemerin receptor CMKLR1 is a functional receptor for amyloid- $\beta$ peptide. J Alzheimers Dis 43: 227-242, 2015.

22. Lehrke M, Becker A, Greif M, Stark R, Laubender RP, von Ziegler F, Lebherz C, Tittus J, Reiser M, Becker C, et al: Chemerin is associated with markers of inflammation and components of the metabolic syndrome but does not predict coronary atherosclerosis. Eur J Endocrinol 161: 339-344, 2009.

23. Zhuang X, Sun F, Li L, Jiang D, Li X, Sun A, Pan Z, Lou N, Zhang L and Lou F: Therapeutic effect of metformin on chemerin in non-obese patients with non-alcoholic fatty liver disease (NAFLD). Clin Lab 61: 1409-1414, 2015.

24. Stejskal D, Karpisek M, Hanulova Z and Svestak M: Chemerin is an independent marker of the metabolic syndrome in a Caucasian population - a pilot study. Biomed Pap Med Fac Univ Palacky Olomouc Czech Repub 152: 217-221, 2008.

This work is licensed under a Creative Commons Attribution-NonCommercial-NoDerivatives 4.0 International (CC BY-NC-ND 4.0) License. 